\title{
DIRECT ESTIMATION \\ OF FINE AND COARSE MODE PARTICLE PARAMETERS FROM MULTIWAVELENGTH LIDAR MEASUREMENTS
}

\author{
Alexei Kolgotin ${ }^{1}$, Mikhail Korenskiy ${ }^{1}$, Igor Veselovskii ${ }^{1}$, David N. Whiteman ${ }^{2}$ \\ ${ }^{1}$ Physics Instrumentation Center, Troitsk, Moscow Region, 142190, Russia, \\ E-mails: alexeift@yahoo.com, miklekor@pic.troitsk.ru, igorv@pic.troitsk.ru \\ ${ }^{2}$ NASA Goddard Space Flight Center, Greenbelt, MD 20771, USA, \\ E-mail: david.n.whiteman@nasa.gov
}

\begin{abstract}
An approach for the direct estimation (DE) of particle parameters in the fine and coarse mode from multiwavelength lidar measurements is presented. Particle size distributions in both modes are approximated by rectangular functions, so the particle density is estimated directly without solving the inverse problem. The numerical simulation demonstrates that the particle volume in both modes can be estimated from $3 \beta+2 \alpha$ lidar measurements with uncertainty of $\sim 25 \%$ for a wide range of size distributions. The technique developed was applied to the observations of NASA GSFC Raman lidar. Comparison of the results obtained with DE and regularization approach applied to the same set of data demonstrates agreement between these two techniques.
\end{abstract}

\section{INTRODUCTION}

Retrieval of particle microphysical properties from multiwavelength Mie-Raman lidar observations has become a widely discussed problem during the last decade. Numerous approaches were suggested for inversion of lidar data to particle parameters [e.g.1-3]. However, even application of complicated mathematical methods does not guarantee that the acceptable solutions can be found without additional constraints on solution space. The main reason is that the inverse problem is strongly underdetermined: from lidar measurements normally only three backscattering $(\beta)$ and two extinction $(\alpha)$ coefficients are available in a limited spectral interval $355-1064 \mathrm{~nm}$ (so called $3 \beta+2 \alpha$ set). All reported results are related mainly to the retrieval of total particle volume and effective radius attributed also to the total size distribution. At the same time in many applications it is very important to separate the contributions of the fine and the coarse modes. Here we present an approach for direct estimation of the fine and the coarse mode parameters without solving the inverse problem.

\section{METHODOLOGY}

The particle extinction and backscattering coefficients are related to the volume size distribution $v(r)$ through the integral equation

$$
\int_{r_{\min }}^{r_{\max }} k_{i}^{V}(m, r) v(r) d r=g_{i}
$$

where $g_{i}$ are lidar measured data ( $\alpha$ or $\left.\beta\right)$ at different wavelengths, $r$ is the particle radius, $m=m_{R}$ - $i m_{I}$ - complex refractive index (CRI) and $k_{i}^{V}(m, r)$ are the kernel functions calculated on a base of Mie theory. Eq. (1) can be also rewritten for number $n(r)$ and surface $s(r)$ density using the corresponding kernel functions $k_{i}^{N}(m, r)$ and $k_{i}^{S}(m, r)$.

The size distribution $v(r)$ can be approximated by a rectangular function on some interval $\left[r_{\text {min }}, r_{\text {max }}\right]$ with maximal value $\frac{V}{r_{\text {max }}-r_{\text {min }}}$ such that for $g_{i}$ data

$$
\frac{V_{i}}{r_{\min }-r_{\max }} \int_{r_{\min }}^{r_{\max }} k_{i}^{V}(m, r) d r=g_{i}
$$

Then the total volume can be found as

$$
V_{i}=\frac{g_{i}\left(r_{\max }-r_{\min }\right)}{K_{i}^{V}\left(m, r_{\min }, r_{\max }\right)}
$$

where

$$
K_{i}^{V}\left(m, r_{\min }, r_{\max }\right)=\int_{r_{\min }}^{r_{\max }} k_{i}^{V}(m, r) d r
$$

is the integrated kernel function.

The volume found in this way is not unique and depends on the choice of the interval $\left[r_{\min }, r_{\max }\right]$ and refractive index. So if the volume is obtained by using several optical data, it is obvious that for "true" solution the results obtained with different $g_{i}$ should coincide.

Eq. (3) is an algebraic equation containing five unknown parameters $V, m_{R}, m_{I}, r_{\min }, r_{\max }$. In contrast to eq. (1) it is parametric problem. In principle, the eq. (3) can be solved if $3 \beta+2 \alpha$ meas- 
urements are available. To solve this equation we predefine the set of input parameters $r_{\min }, r_{\max }, m_{R}$, $m_{I}$ the same way as we did in [2].

If we have $\mathrm{N}_{0}$ data $\left(\mathrm{N}_{0}=5\right.$ for $3 \beta+2 \alpha$ set $)$ we obtain $\mathrm{N}_{0}$ solutions $V_{1}, \ldots, V_{\mathrm{N}_{0}}$ for each set of input parameters. The mean value of volume $\bar{V}$ is

$$
\bar{V}=\frac{1}{\mathrm{~N}_{\mathrm{o}}} \sum_{i=1}^{\mathrm{N}_{\mathrm{o}}} V_{i}
$$

and the relative deviation from mean value $\Delta_{V}$ is

$$
\Delta_{V}=\frac{100 \%}{\bar{V}} \sqrt{\frac{1}{\mathrm{~N}_{\mathrm{o}}} \sum_{i=1}^{\mathrm{N}_{\mathrm{o}}}\left(V_{i}-\bar{V}\right)^{2}}
$$

The deviation $\Delta_{V}$ can be treated as discrepancy in solution space $\rho_{V}=\Delta_{V}$. Since all solutions $V_{1}, \ldots$, $V_{\mathrm{N}_{0}}$ should coincide we should look for minimum of discrepancy $\rho_{V}$ to determine input parameters $m_{R}, m_{l}, r_{\min }, r_{\max }$.

We can also introduce the discrepancy $\rho_{g}$ in the optical data space

$$
\rho_{g}=\sqrt{\frac{1}{\mathrm{~N}_{\mathrm{o}}} \sum_{i=1}^{\mathrm{N}_{\mathrm{o}}}\left(\frac{g_{i}-\bar{g}_{i}}{g_{i}}\right)^{2}}
$$

where back-calculated optical data are found as

$$
\bar{g}_{i}=\frac{\bar{V}}{r_{\max }-r_{\min }} K_{i}^{V}\left(m, r_{\min }, r_{\max }\right), i=1, \ldots, \mathrm{N}_{\mathrm{o}}
$$

The numerical simulations performed demonstrate, that best results are obtained when both discrepancies are used, so in our algorithm we minimize the sum

$$
\rho=\rho_{g}+\rho_{V}
$$

Minimization of discrepancy provides the estimation of particle volume.

Similar consideration can be performed for the particle number $n(r)$ and surface $s(r)$ concentrations and effective radius can be calculated as $r_{\text {eff }}=3 \frac{V}{S}$.

The approach presented can be extended to the case of bimodal PSD. The $v(r)$ is represented by a combination of two independent distributions related to the fine $v^{f}(r)$ and course $v^{c}(r)$ modes

$$
v(r)=v^{f}(r)+v^{c}(r)
$$

The size distributions for the fine and coarse mode can be approximated by a rectangular functions on interval $\left[r_{\min }^{f}, r_{\max }^{f}\right]$ and $\left[r_{\min }^{c}, r_{\max }^{c}\right]$ respectively with maximal values $\frac{V^{f}}{r_{\max }^{f}-r_{\min }^{f}}$ and $\frac{V^{c}}{r_{\max }^{c}-r_{\min }^{c}}$, as shown in fig.1.
For a bimodal size distribution the eq.(3) is transformed to :

$$
V^{f} \frac{K_{i}^{V}\left(m, r_{\min }^{f}, r_{\max }^{f}\right)}{r_{\min }^{f}-r_{\max }^{f}}+V^{c} \frac{K_{i}^{V}\left(m, r_{\min }^{c}, r_{\max }^{c}\right)}{r_{\min }^{c}-r_{\max }^{c}}=g_{i}
$$

and for every pair of $g_{i}, g_{j}$ data the volumes $V^{f}, V^{c}$ can be found as:

$$
\left\{\begin{array}{l}
V_{j, i}^{f}=\frac{A_{i}^{V^{c}}(m) g_{j}-A_{j}^{V^{c}}(m) g_{i}}{A_{j}^{V^{f}}(m) A_{i}^{V^{c}}(m)-A_{j}^{V^{c}}(m) A_{i}^{V^{f}}(m)} \\
V_{j, i}^{c}=\frac{A_{j}^{V^{f}}(m) g_{i}-A_{i}^{V^{f}}(m) g_{j}}{A_{j}^{V^{f}}(m) A_{i}^{V^{c}}(m)-A_{j}^{V^{c}}(m) A_{i}^{V^{f}}(m)}
\end{array}\right.
$$

where

$$
\begin{aligned}
& A_{l}^{V^{c}}(m)=\frac{K_{l}^{V}\left(m, r_{\text {min }}^{c}, r_{\text {max }}^{c}\right)}{r_{\text {min }}^{c}-r_{\text {max }}^{c}} ; \\
& A_{l}^{V^{f}}(m)=\frac{K_{l}^{V}\left(m, r_{\text {min }}^{f}, r_{\text {max }}^{f}\right)}{r_{\text {min }}^{f}-r_{\text {max }}^{f}}
\end{aligned}
$$

and $l=i, j$. Thus for 5 input data we have ten equations (11) for volume density. To solve these we minimize the discrepancy (9) as was explained above. Since solutions $V^{f}, V^{c}$ are expressed explicitly we call this approach the direct estimation (DE). The important distinction from regularization retrieval is that $\mathrm{DE}$ does not contain an ill-posedness.

We should recall, that problem (12) contains eight unknown parameters $V^{f}, V^{c}, m_{R}, m_{l}, r_{\min }{ }^{f}$, $r_{\max }{ }^{f}, r_{\min }{ }^{c}, r_{\max }{ }^{c}$, while only five input data are available, so the problem is strongly underdetermined. Such situation is typical, when lidar measurements are inverted to particle parameters: instead a unique solution a family of solutions is obtained. The limitations we put on the "search space" of predefined parameters work as a constrain and help to decrease the number of solutions in the family. The found solutions are ranged in accordance with discrepancy $\rho$. Normally we are searching for $m_{\mathrm{R}}$ and $m_{\mathrm{I}}$ inside the intervals 1.3-1.6 and 0-0.015 respectively. The particle radii are taken on interval $0.075-6.0 \mu \mathrm{m}$, so the total number of parameters sets considered is of order $10^{5}$. Usually we average $0.1 \%$ of total amount of solutions.

\section{NUMERICAL SIMULATION}

A numerical simulation was performed to estimate the uncertainties of DE approach for retrieval the particle volume, effective radius and refractive index. The particle bimodal size distribution was represented as: 


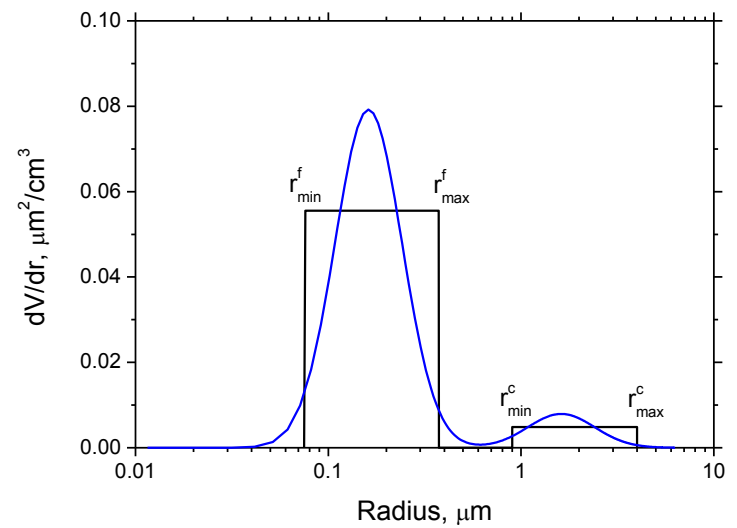

Fig.1. The model (blue) and retrieved (black) with DE approach the particle volume size distribution.

$$
\frac{d n(r)}{d \ln (r)}=\sum_{i=f, c} \frac{N^{i}}{(2 \pi)^{1 / 2} \cdot \ln \sigma^{i}} \cdot \exp \left[\frac{\left(\ln r-\ln r_{0}^{i}\right)^{2}}{2\left(\ln \sigma^{i}\right)^{2}}\right]
$$

Here $N^{i}$ is the total particle number of the $i$-th mode, $r_{0}{ }^{i}$ describes the mode radius and $\ln \sigma^{i}$ is the dispersion. The index $i=f, c$ corresponds to the fine and coarse mode, respectively. For all computations the values $r_{0}^{f}=0.1 \mu \mathrm{m}, r_{0}{ }^{c}=1 \mu \mathrm{m}$ and $\ln \sigma^{f, c}=0.4$ were used and the complex refractive index (CRI) was assumed to be the same for both modes. To account for different PSDs, three ratios $N^{\mathrm{c}} / N^{f}=10^{-2}, 10^{-3}, 10^{-4}$ were considered. For ratio $10^{-2}$, the coarse mode is predominant, while for ratio $10^{-4}$ the main part of the volume is contained in the fine mode.

The $3 \beta+2 \alpha$ lidar measurements are not very sensitive to the coarse mode contribution when the fine mode is predominant, because the maximal wavelength available is only $1064 \mathrm{~nm}$. This is illustrated by Fig.2, showing the contribution of the coarse mode to the optical data for different ratios $V^{c} / V^{t}$, where $V^{t}=V^{f}+V^{c}$. Keeping in mind that typical uncertainty for optical data is $5-10 \%$, we cannot expect retrieval of the coarse mode parameters for $V^{c} / V^{t}<0.1$. Basing on results of numerous simulations we introduced the limitations in the algorithm: for retrieved $V^{c} / V^{t}<0.2$ the parameters of the coarse mode are not considered.

Fig.3a shows the uncertainties of estimation of $V^{f}, \quad V^{c}, \quad V^{t}$ for these three $N^{c} / N^{f}$ ratios. For $N^{c} / N^{f}=10^{-4}$ the contribution of the coarse mode is too low, so the program doesn't consider it. For the intermediate case, when contributions of the fine and coarse mode are comparable, the uncertainty of volume estimation is about $20 \%$. For the coarse mode predominance the errors for $V^{f}$ are still about $20 \%$, while for $V^{C}$ it is below $5 \%$. Errors for effective radius are higher and may reach $40 \%$ for $N^{c} / N^{f}=10^{-3}$ as shown in Fig.2b. The un- certainty of estimation of the real part of refractive index is estimated as \pm 0.05 .

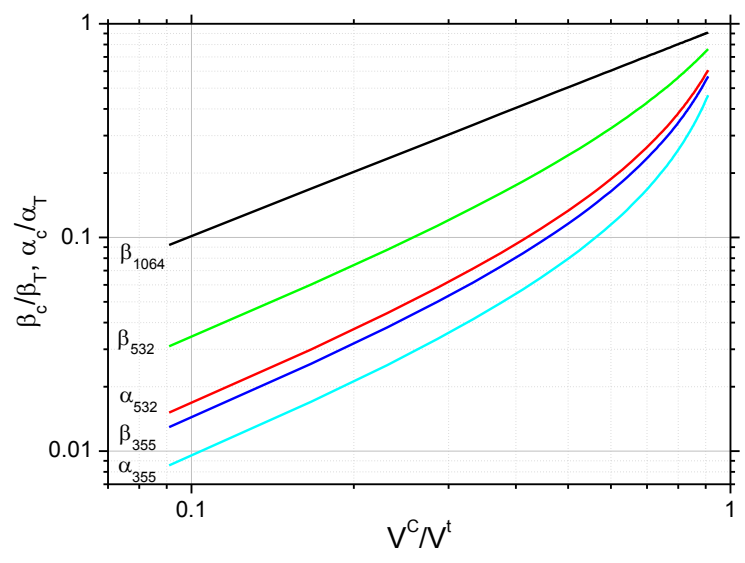

Fig.2. Contribution of the coarse mode to the optical data depending on the coarse mode volume fraction. Computations were performed for $\mathrm{m}=1.45-\mathrm{i} 0.005$.
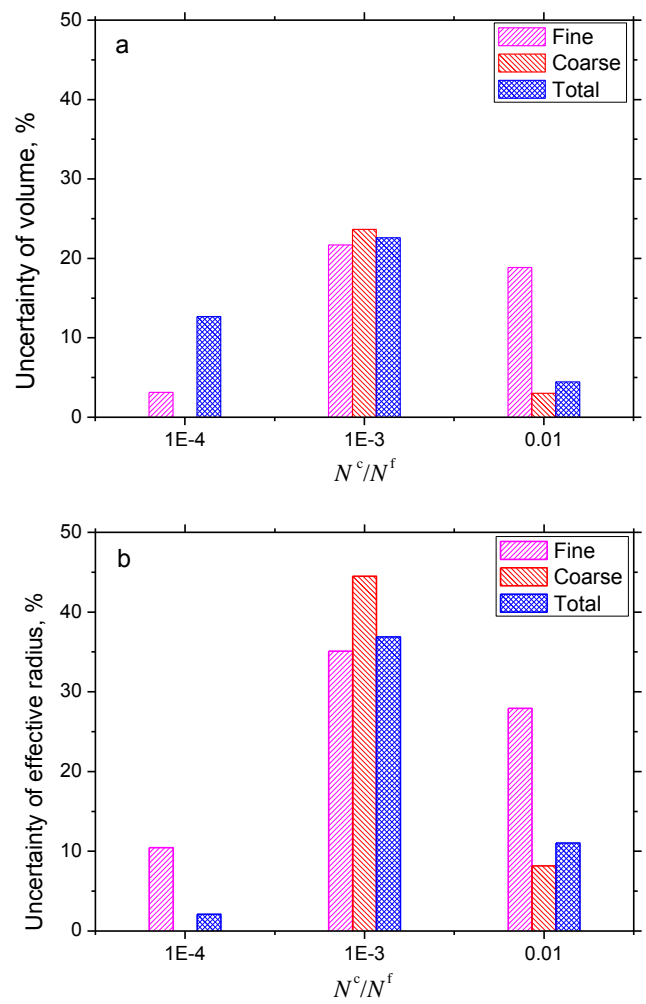

Fig.3. Uncertainties of (a) volume and (b) effective radius retrieval for different ratios of particle number density in the coarse and fine mode. Simulations were performed for $\mathrm{m}=1.45-\mathrm{i} 0.005$.

\section{APPLICATION TO LIDAR MEASUREMENTS}

To test the developed algorithm the measurements performed by NASA GSFC multiwavelength Raman lidar [4] on 22 August 2006 were used. The measured $3 \beta+2 \alpha$ lidar data were inverted to the particle microphysics by using regularization [2] and DE algorithms. Fig.4.shows the vertical profiles of the particle volume density obtained with two approaches. 
Contribution of the coarse mode for this day was too low, so DE algorithm automatically put it to zero, as described in previous section. The volume densities $V^{t}$ obtained with the two approaches are close, while the effective radii differ significantly for the heights above $1000 \mathrm{~m}$. DE leads to higher values of $r_{\text {eff }}$ and to lower values of the real part of CRI above $1000 \mathrm{~m}$ height, as shown in Fig.5. This agrees with results of numerical simulations, showing that DE approach is most suitable for estimation of particle volume.

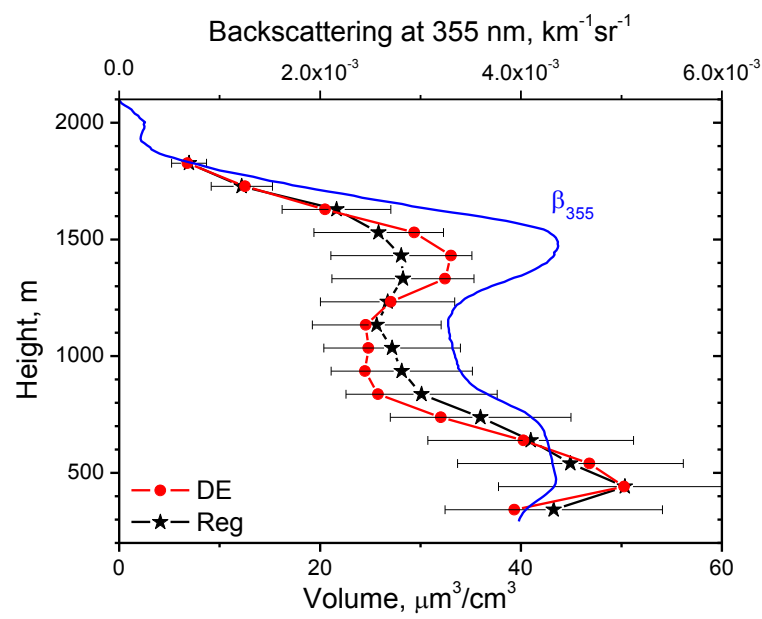

Fig.4. Vertical profiles of the volume density obtained with regularization and DE approach. Blue solid line shows the backscattering coefficient at $355 \mathrm{~nm}$.

\section{CONCLUSION}

An approach for estimation of the particle volume in the fine and in the coarse mode is presented. The numerical simulations performed demonstrate that particle volume in both modes can be derived with uncertainty about $25 \%$ for a wide range of PSDs. To evaluate this approach $\mathrm{DE}$ and regularization algorithms were applied to the same multiwavelength measurements. The comparison demonstrates an agreement between these two approaches. In all measurements considered the fine mode was predominant and contribution of the coarse mode was too small to be retrieved with the DE technique. In the future we plan to consider desert dust measurements, characterized by strong coarse mode. To treat the dust particles the spheroids mode will be incorporated in the algorithm in the nearest future.
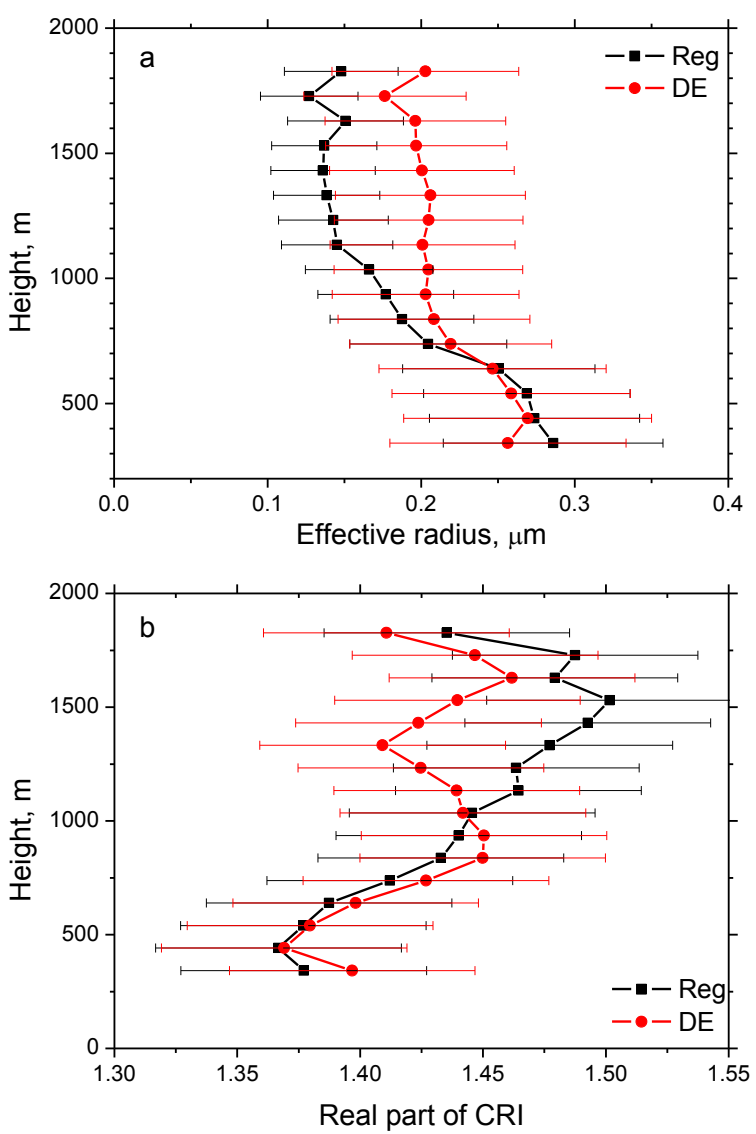

Fig.5. Vertical profiles of (a) effective radius and (b) real part of CRI obtained with regularization and DE approach.

\section{REFERENCES}

[1] Ansmann, A. and Müller, D.: Lidar and atmospheric aerosol particles, in "Lidar. RangeResolved Optical Remote Sensing of the Atmosphere", Weitkamp, C. ed., Springer, New York, 2005, pp. 105-141.

[2] Veselovskii I., et al.,: Inversion with regularization for the retrieval of tropospheric aerosol parameters from multiwavelength lidar sounding, Appl. Opt., 41, pp. 3685 - 3699, 2002.

[3] Chemyakin E., et al,: "Arrange \& average" algorithm for the retrieval of aerosols parameters from multiwavelength HSRL/Raman lidar data, Appl. Opt., 41, pp. 3685 - 3699, 2014.

[4] Veselovskii I., et al.: Demonstration of aerosol property profiling by multi-wavelength lidar under varying relative humidity conditions, J. of Atmospheric and Oceanic Tech. 26, 1543-1557, 2009. 